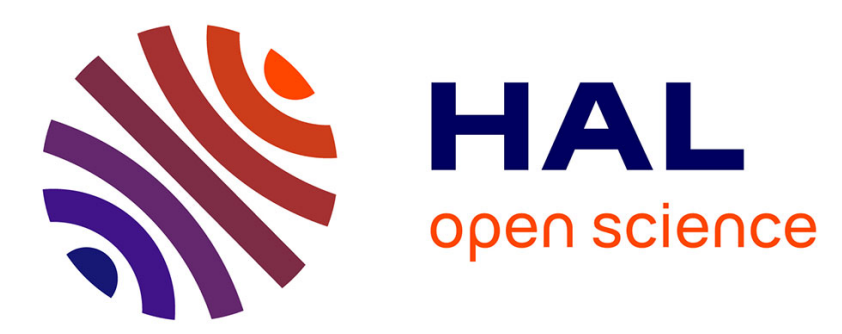

\title{
Conjugate observations of quasi-periodic emissions by Cluster and DEMETER spacecraft
}

F. Němec, O. Santolík, Michel Parrot, J. S. Pickett, M. Hayosh, Nicole

Cornilleau-Wehrlin

\section{- To cite this version:}

F. Němec, O. Santolík, Michel Parrot, J. S. Pickett, M. Hayosh, et al.. Conjugate observations of quasi-periodic emissions by Cluster and DEMETER spacecraft. Journal of Geophysical Research Space Physics, 2013, 118 (1), pp.198-208. 10.1029/2012JA018380 . insu-01289740

\section{HAL Id: insu-01289740 \\ https://hal-insu.archives-ouvertes.fr/insu-01289740}

Submitted on 20 May 2016

HAL is a multi-disciplinary open access archive for the deposit and dissemination of scientific research documents, whether they are published or not. The documents may come from teaching and research institutions in France or abroad, or from public or private research centers.
L'archive ouverte pluridisciplinaire HAL, est destinée au dépôt et à la diffusion de documents scientifiques de niveau recherche, publiés ou non, émanant des établissements d'enseignement et de recherche français ou étrangers, des laboratoires publics ou privés. 


\title{
Conjugate observations of quasi-periodic emissions by Cluster and DEMETER spacecraft
}

\author{
F. Němec, ${ }^{1}$ O. Santolík, ${ }^{2,1}$ M. Parrot,${ }^{3}$ J. S. Pickett, ${ }^{4}$ M. Hayosh, ${ }^{2}$ \\ and N. Cornilleau-Wehrlin ${ }^{5,6}$ \\ Received 8 October 2012; revised 12 November 2012; accepted 13 November 2012; published 31 January 2013.
}

[1] Quasi-periodic (QP) emissions are electromagnetic emissions at frequencies of about $0.5-4 \mathrm{kHz}$ that are characterized by a periodic time modulation of the wave intensity. Typical periods of this modulation are on the order of minutes. We present a case study of a large-scale long-lasting QP event observed simultaneously on board the DEMETER (Detection of Electro-Magnetic Emissions Transmitted from Earthquake Regions) and the Cluster spacecraft. The measurements by the Wide-Band Data instrument on board the Cluster spacecraft enabled us to obtain high-resolution frequency-time spectrograms of the event close to the equatorial region over a large range of radial distances, while the measurements by the STAFF-SA instrument allowed us to perform a detailed wave analysis. Conjugate observations by the DEMETER spacecraft have been used to estimate the spatial and temporal extent of the emissions. The analyzed QP event lasted as long as $5 \mathrm{~h}$ and it spanned over the $L$-shells from about 1.5 to 5.5. Simultaneous observations of the same event by DEMETER and Cluster show that the same QP modulation of the wave intensity is observed at the same time at very different locations in the inner magnetosphere. ULF magnetic field fluctuations with a period roughly comparable to, but somewhat larger than the period of the QP modulation were detected by the fluxgate magnetometers instrument on board the Cluster spacecraft near the equatorial region, suggesting these are likely to be related to the QP generation. Results of a detailed wave analysis show that the QP emissions detected by Cluster propagate unducted, with oblique wave normal angles at higher geomagnetic latitudes.

Citation: Němec, F., O. Santolík, M. Parrot, J. S. Pickett, M. Hayosh, and N. Cornilleau-Wehrlin (2013), Conjugate observations of quasi-periodic emissions by Cluster and DEMETER spacecraft, J. Geophys. Res. Space Physics, 118, 198-208, doi:10.1029/2012JA018380.

\section{Introduction}

[2] Quasi-periodic (QP) emissions are ELF/VLF electromagnetic emissions observed in the magnetosphere at frequencies of about $0.5-4 \mathrm{kHz}$, exhibiting a periodic time modulation of wave intensity. The periods of this modulation are generally above $10 \mathrm{~s}$, in contrast with $4-6 \mathrm{~s}$ periods of periodic emissions due to ducted whistler mode waves

\footnotetext{
${ }^{1}$ Faculty of Mathematics and Physics, Charles University in Prague, Prague, Czech Republic.

${ }^{2}$ Institute of Atmospheric Physics, Academy of Sciences of the Czech Republic, Prague, Czech Republic.

${ }^{3}$ Laboratoire de Physique et Chimie de 1'Environnement et de l'Espace, Centre National de la Recherche Scientifique, Orléans, France.

${ }^{4}$ Department of Physics and Astronomy, University of Iowa, Iowa City, Iowa, USA.

${ }^{5}$ Laboratoire de Physique des Plasmas, Ecole Polytechnique, CNRS, Palaiseau, France.

${ }^{6}$ LESIA, Observatoire de Meudon, Meudon, France.

Corresponding author: F. Němec, Faculty of Mathematics and Physics, Charles University in Prague, Prague, Czech Republic.

(frantisek.nemec@gmail.com)

(C)2012. American Geophysical Union. All Rights Reserved. 2169-9380/13/2012JA018380
}

echoing along geomagnetic-field-aligned paths between opposite hemispheres [Helliwell, 1965; Carson et al., 1965; Sazhin and Hayakawa, 1994; Smith et al., 1998]. The QP emissions appear to be essentially a dayside phenomenon [Ho, 1973; Kimura, 1974; Morrison et al., 1994; Engebretson et al., 2004] and they can be further classified, from ground observations, as QP emissions type 1 and QP emissions type 2, depending on whether or not correlated coincident ULF geomagnetic pulsations are detected [Kitamura et al., 1969; Sato et al., 1974].

[3] Quasi-periodic events of type 1, which are closely correlated with coincident geomagnetic pulsations, are likely to be caused by quasi-periodic fluctuations of resonant conditions and wave growth in the wave generation region, governed by the ULF magnetic field oscillations [Kimura, 1974; Chen, 1974; Sato and Fukunishi, 1981; Sazhin, 1987; Watt et al., 2011]. This wave generation region seems to be located close to the equatorial plane in the outer magnetosphere [Sato and Kokubun, 1980; Sato and Fukunishi, 1981; Morrison, 1990]. A possible importance of highlatitude (nonequatorial) magnetic field minima near the magnetopause for the frequency spectra of the observed QP emissions was discussed by Alford et al. [1996]. Tixier and Cornilleau-Wehrlin [1986] have shown that the classification 
between QP emissions of type 1 and type 2 is not so obvious in the space observations as on the ground, and they suggested that both types of QP emissions might have the same generation mechanism. Quasi-periodic pulsations in precipitating electron fluxes related to the generation of QP emissions were considered by Coronity and Kennel [1970] and reported by Gendrin et al. [1970a, 1970b]. A generation of quasi-periodic magnetic pulsations in the ionosphere due to the quasiperiodic modulation of precipitating electron fluxes has been also considered [Bell, 1976; Sato and Kukubun, 1981; Sato and Matsudo, 1986]. These magnetic pulsations can be subsequently detected on the ground and explain some of the observed magnetic field fluctuations related to the QP emissions [Sato and Fukunishi, 1981]. QP events with a time duration longer than $1 \mathrm{~h}$ have been reported using single ground stations [Lanzerotti et al., 1986]. Nevertheless, they can last for many hours at the same local time, being observed successively by several ground stations spaced in longitude as each of them comes into the active region due to the Earth's rotation [Smith et al., 1991]. The occurrence statistics show that QP emissions favor quiet geomagnetic conditions [Ho, 1973]. A case study of QP emissions detected on board the Freja and the Magion 5 satellites was presented by Pasmanik et al. [2004], along with possible scenarios for their formation.

[4] We present a case study of QP emissions observed simultaneously on board the DEMETER (Detection of Electro-Magnetic Emissions Transmitted from Earthquake Regions) and the Cluster spacecraft. Conjugate observations of the same event at several different points enable us to distinguish between spatial and temporal variations. Moreover, multicomponent measurements of the electromagnetic field allow us to perform a detailed wave analysis of the emissions. Section 2 describes the satellites and the data used in the study. The results that we have obtained are presented in section 3 and discussed in section 4. Finally, section 5 contains a brief summary.

\section{DEMETER and Cluster Spacecraft}

[5] DEMETER was a low-altitude satellite that operated from 2004 to 2010. It had a circular polar orbit with an altitude of about $660 \mathrm{~km}$. The orbit was nearly Sun-synchronous, i.e., the spacecraft was always located close to the local noon (about 10:30 LT) or the local midnight (about 22:30 LT). Among all the instruments on board, only the electric field instrument will be used in the present study [Berthelier et al., 2006]. In the VLF range this instrument provides us with a power spectrum of one electric field component measured continuously at geomagnetic latitudes from $-65^{\circ}$ to $65^{\circ}$. The frequency resolution of the spectrum is $19.53 \mathrm{~Hz}$ and the time resolution is 2.048 or $0.512 \mathrm{~s}$, depending on the mode of operation.

[6] The four Cluster spacecraft, operated by the European Space Agency, move in a close formation along an elliptical orbit with a perigee of about $1.5 R_{\mathrm{E}}$ at geomagnetic latitudes of about $40^{\circ}$ and cross the geomagnetic equator at a radial distance of approximately $6 R_{\mathrm{E}}$ (because the spacecraft orbits changed over the duration of the mission, these are approximate parameters for the time period of interest). The WideBand Data (WBD) plasma wave investigation instruments on board the Cluster spacecraft were designed to provide high-resolution measurements of both electric and magnetic fields [Gurnett et al., 1997]. During the event investigated in this study, the WBD instrument operated in a mode which cycled between obtaining waveforms of one electric field component measured in the spin plane of the spacecraft for $42 \mathrm{~s}$ and waveforms of one magnetic field component along the spin axis for $10 \mathrm{~s}$. In addition to the WBD instruments, the STAFF-SA (Spatio-Temporal Analysis of Field Fluctuations - Spectrum Analyzer) instruments acquired information about power-spectral densities, mutual phases, and coherence relations of three orthogonal magnetic field components and two electric field components [CornilleauWehrlin et al., 1997, 2003]. The analysis is made on 27 logarithmically-spaced frequency channels between 8 and $4 \mathrm{kHz}$. The data are preanalyzed on board with the time resolution for the studied event of $1 \mathrm{~s}$ (for the power-spectral densities) and $4 \mathrm{~s}$ (for the phases and the coherence), and Hermitian spectral matrices $5 \times 5$ are constructed, one matrix per frequency channel and time interval. These can be used to determine detailed wave properties [see, e.g., Santolik et al., 2003, and the references therein]. Finally, the Cluster spacecraft are equipped with fluxgate magnetometers (FGM) [Balogh et al., 1997, 2001] that provide us with spin-averaged measurements of three components of the ambient magnetic field with a sampling frequency of $0.25 \mathrm{~Hz}$.

\section{Results}

[7] A search for possible conjunctions between the DEMETER and the Cluster spacecraft during the time intervals when at least one of the WBD instruments was active revealed a QP event observed simultaneously by DEMETER and Cluster. According to the DEMETER data, the event occurred on 13 April 2010 between about 04:30 UT and 09:15 UT. The WBD instruments on board the Cluster spacecraft were active only on Cluster 2 and Cluster 4 and only for a part of the time interval of interest. As soon as the WBD instruments turned on at about 07:52 UT on Cluster 2 and at about 07:59 UT on Cluster 4, they started to observe the QP emissions and observed them until about 09:25 UT, when they gradually faded. Unfortunately, the electric field antenna on Cluster 2 was not functioning correctly beginning about 07:58 UT, so that only the $10 \mathrm{~s}$ long snippets of the magnetic field data are available. Although these cannot be effectively used for the analysis, they allow us to confirm the presence of the QP emissions. In addition to the WBD instrument, the STAFF-SA instruments were active for a longer time period on board Cluster 4 and Cluster 2. These have a poor frequency resolution at higher frequencies, and their sensitivity to the QP emissions is therefore significantly lower. They observed the QP emissions only between about 08:10 UT and 09:00 UT. It should be noted that the geomagnetic activity on 13 April 2010 was extremely low (daily Kp sum 5o), but it had been higher one day before (daily Kp sum 22+).

[8] Figure 1 shows frequency-time spectrograms of the power spectral density of electric field fluctuations corresponding to the QP event measured by the DEMETER spacecraft. Figures $1 \mathrm{a}-1 \mathrm{~d}$ correspond to one DEMETER half-orbit, i.e., to about $35 \mathrm{~min}$ of data spanning from about $65^{\circ}$ of geomagnetic latitude down to about $-65^{\circ}$ of 
(a)

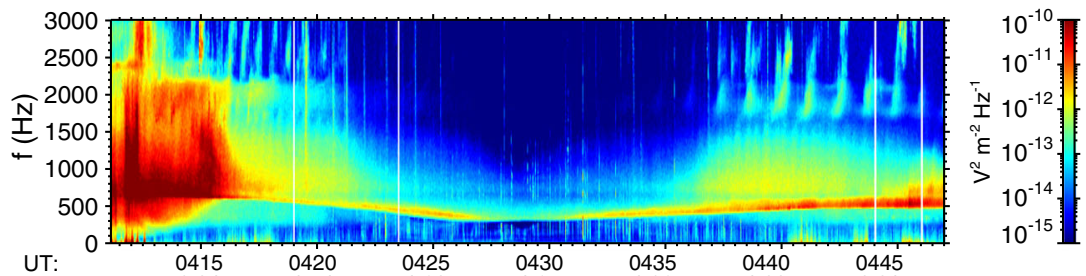

(b)

(c)

(d)

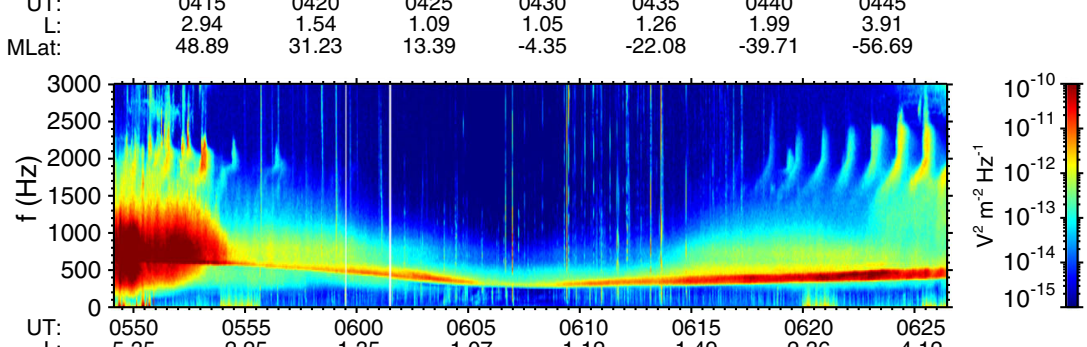$$
\begin{array}{rcccccccc}
\text { L: } & 5.35 & 2.25 & 1.35 & 1.07 & 1.12 & 1.49 & 2.36 & 4.12 \\
\text { MLat: } & 60.00 & 43.57 & 26.44 & 8.98 & -8.50 & -25.94 & -42.98 & -58.97
\end{array}
$$
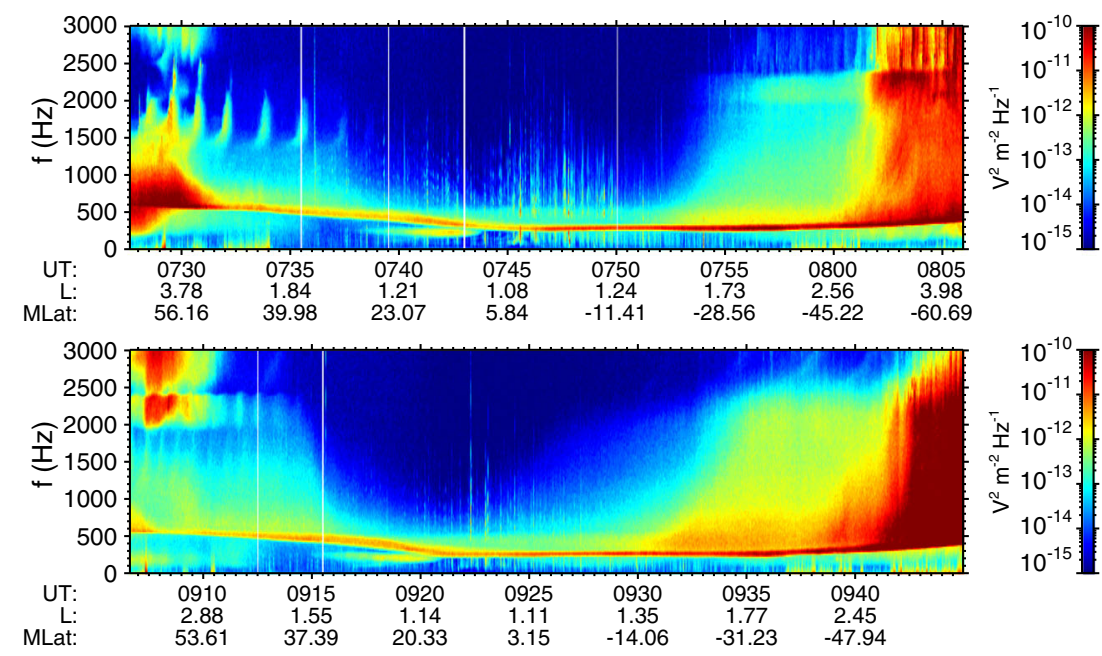

Figure 1. Frequency-time spectrograms of power spectral density of electric field fluctuations measured by DEMETER on 13 April 2010. Each of the four panels corresponds to one DEMETER half-orbit, i.e., to approximately $35 \mathrm{~min}$ of data spanning from about $65^{\circ}$ of geomagnetic latitude down to $-65^{\circ}$ of geomagnetic latitude. QP emissions can be clearly distinguished in the right-hand parts of Figures $1 \mathrm{a}$ and $1 \mathrm{~b}$ and left-hand parts of panels Figures 1c and 1d. Moreover, somewhat less pronounced QP emissions are seen in the left-hand part of panel Figure 1b.

geomagnetic latitude. The emissions were observed during "down-going" half-orbits, i.e., close to the local noon. The frequency range spans from 0 to $3 \mathrm{kHz}$ and the universal time, the $L$-value, and the geomagnetic latitude are provided on the abscissa axis. QP emissions can be clearly distinguished in the right-hand parts of Figures $1 \mathrm{a}$ and $1 \mathrm{~b}$ and the left-hand parts of Figures 1c and 1d. Moreover, somewhat less pronounced QP emissions are seen in the lefthand part of Figure 1b. This shows that the occurrence of QP emissions as observed by the DEMETER spacecraft is limited to $L$-shells from about 1.5 up to 6 . No similar emissions were observed during the "up-going" half-orbits, i.e., close to the local midnight. It should be noted that QP wave activity was observed also several orbits before and several orbits after the four orbits plotted in Figure 1. Nevertheless, it seems - at least as far as one can say from a limited coverage of the DEMETER data - that this surrounding activity was separated by calmer intervals, so that it is not clear if it should be attributed to the QP event under study or to another QP event starting shortly before/after. However, this does not concern the topic of the paper, as it affects only the estimate of the total time duration of the event.

[9] Frequency-time spectrograms of power spectral density of electric field fluctuations measured by the WBD instrument on board the Cluster 4 spacecraft between 08:00 UT and 09:30 UT are shown in Figure 2. Figures 2a and $2 \mathrm{~b}$ correspond to $45 \mathrm{~min}$ of data. Cyclic $42 \mathrm{~s}$ data intervals of electric field measurements have been dilated across $10 \mathrm{~s}$ data intervals where the magnetic field had been measured instead throughout. The plotted frequency interval spans again from 0 to $3 \mathrm{kHz}$, and the radial distance, the geomagnetic latitude, and the magnetic local time are shown on the abscissa axis. The QP emissions are well visible since the beginning of the plotted time interval. After 09:00 UT the emissions cease gradually and the QP structure cannot be identified anymore after about 09:25 UT. Individual elements forming the QP emissions can be classified as rising tones [Sato and Fukunishi, 1981]. The appropriate frequency sweep rates $(\mathrm{d} f / \mathrm{d} t)$ are not constant throughout an element, but rather increase as a function of frequency. 
(a)
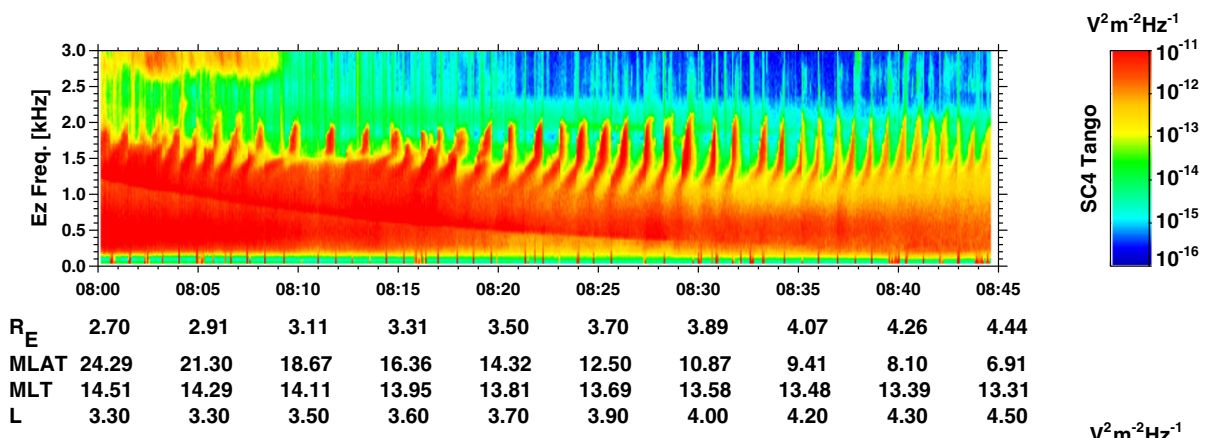

(b)
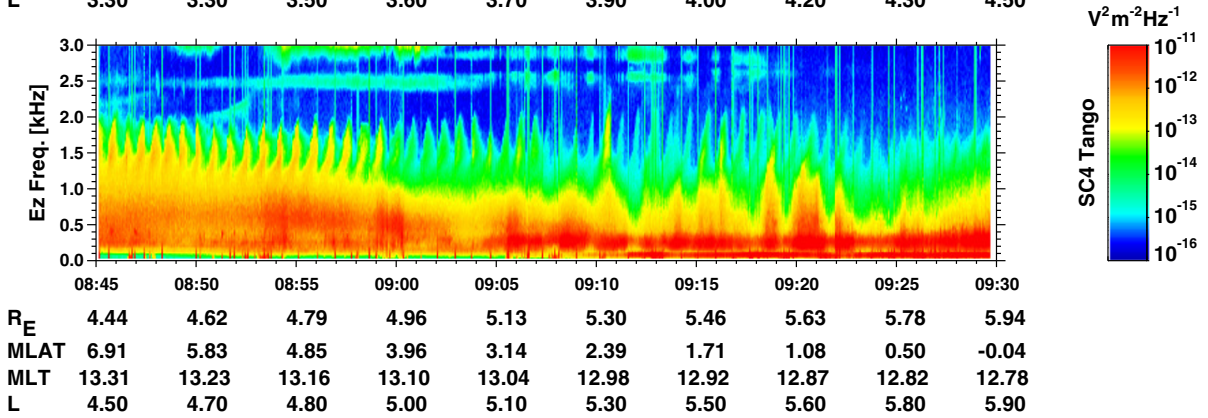

Figure 2. Frequency-time spectrograms of power spectral density of electric field fluctuations measured by the WBD instrument on board Cluster 4 on 13 April 2010 close to the equatorial region at radial distances from about 3 to $6 R_{\mathrm{E}}$. QP emissions are observed for most of the plotted time interval, gradually ceasing toward its end. Each of the two panels corresponds to $45 \mathrm{~min}$ of data.

[10] The period of the QP modulation (i.e., the time separation between individual elements of the emission) determined from the Cluster 4 WBD data is shown in Figure 3. It has been visually determined for five different frequencies, with the results obtained for each of them being shown by a different symbol. It can be seen that after 08:30 UT the time separation between consecutive elements of the QP emission does not depend on the frequency, but varies slightly over the time, from about 40 to $60 \mathrm{~s}$. In the beginning of the event, between about 08:05 UT and 08:20 UT, the time separation of the elements is somewhat larger and it may be as large as nearly $2 \mathrm{~min}$. It should be underlined that although the variation of the time separation of the elements is observed, the time separation does not change abruptly, but rather quite continuously.

[11] When analyzing the time separation between the QP elements, an important question arises, whether the QP structure corresponds to a purely temporal variation or if the satellite location plays any important role, i.e., if the modulation varies from place to place. Generally, this cannot be distinguished by using a single satellite, but simultaneous observations of the same event by two different spacecraft at two different locations enable us to resolve this problem. Figure 4 shows a comparison of frequency-time spectrograms of power spectral density of electric field fluctuations observed by the DEMETER (Figure 4a) and the Cluster 4 spacecraft (Figure $4 b$ ). The plotted time interval and the frequency range is exactly the same in both figures, but the power scale is somewhat different in order to provide a good contrast. Although most of the features in the spectrograms are completely different, one can see that the QP elements observed at frequencies close to $1600 \mathrm{~Hz}$ are rather similar in both panels. Namely, the times and the frequencies of the individual elements observed by DEMETER and Cluster are nearly the same. Their intensity may substantially fluctuate from element to element and it is generally different on the two spacecraft. Taking into account that DEMETER was located at a radial distance of about $1.1 R_{\mathrm{E}}$ and at geomagnetic latitudes from about $50^{\circ}$ to $60^{\circ}$, while Cluster 4 was located close to the geomagnetic equator at a radial distance of about $5.3 R_{\mathrm{E}}$, the agreement in the observed pattern is surprising.

[12] Although high-resolution electric field measurements performed by the WBD instrument are not available for Cluster 2 during this time interval, we can compare the magnetic field data measured by the STAFF-SA instruments on board Cluster 2 and Cluster 4. Locations of Cluster 2 and Cluster 4 spacecraft during the time interval when the QP emissions were observed by the STAFF-SA instruments are shown in Figure 5a by red and blue colors, respectively. Cluster 2 was located at higher geomagnetic latitudes, while Cluster 4 was located closer to the geomagnetic equator. The difference in magnetic local time (MLT) between the two spacecraft was very low, being less than $0.5 \mathrm{~h}$. Magnetic field lines corresponding to $L=4$ and $L=5$ calculated using combined IGRF (International Geomagnetic Reference Field) and Tsyganenko 89 magnetic field models are overplotted. Parts of the orbits corresponding to the $30 \mathrm{~min}$ long time interval between 08:20 UT and 08:50 UT when the QP emissions were exceptionally well pronounced are plotted in bold. The corresponding $L$-values as a function of time are plotted in Figure 5b, using the same color convention as in Figure 5a. Figure 5c shows the power spectral density of magnetic field fluctuations measured by the STAFF-SA instruments during this time interval. We have selected the 23rd frequency band out of the total 27 frequency bands of the instrument, i.e., the frequency band spanning from about $1250 \mathrm{~Hz}$ up to $1575 \mathrm{~Hz}$, which is approximately the central frequency of the observed QP emissions. The values 


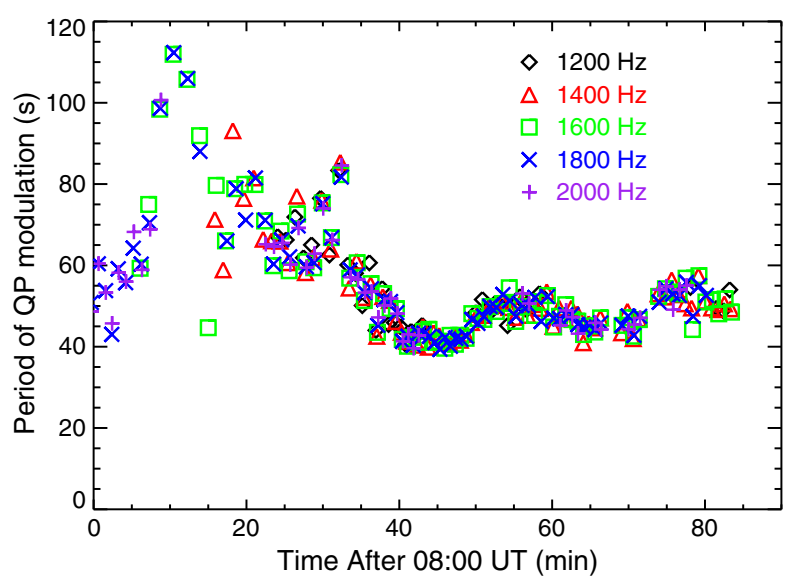

Figure 3. Period of QP modulation (i.e., the time separation of consecutive QP elements) as observed by the Cluster 4 spacecraft on 13 April 2010. Different symbols correspond to different frequencies. observed by Cluster 4 are plotted with a negative sign in order to enable an easy visual comparison with the values observed by Cluster 2 . It can be seen that - although the values of the power spectral density are somewhat different - the intensity modulation observed by the two spacecraft is the same. Namely, the maxima and minima of power spectral density are detected at the same times by Cluster 2 and Cluster 4 . We have used the data from Figure $5 \mathrm{c}$ and a correlation analysis to determine the optimal lag between the two time series, i.e., to determine the average time delay between the signals. The time resolution of the power spectral densities measured by the STAFF-SA instruments is $1 \mathrm{~s}$. Within this time resolution, no time delay between the signals observed by Cluster 2 and Cluster 4 was detected (not shown).

[13] Having shown that the observed QP variations are entirely due to a time modulation, and that the same pattern is observed over a large region of the inner magnetosphere (with possible short time delays that we are not able to exclude with the available data set), it is reasonable to try to estimate the dimensions of the affected region. The obtained

(a)

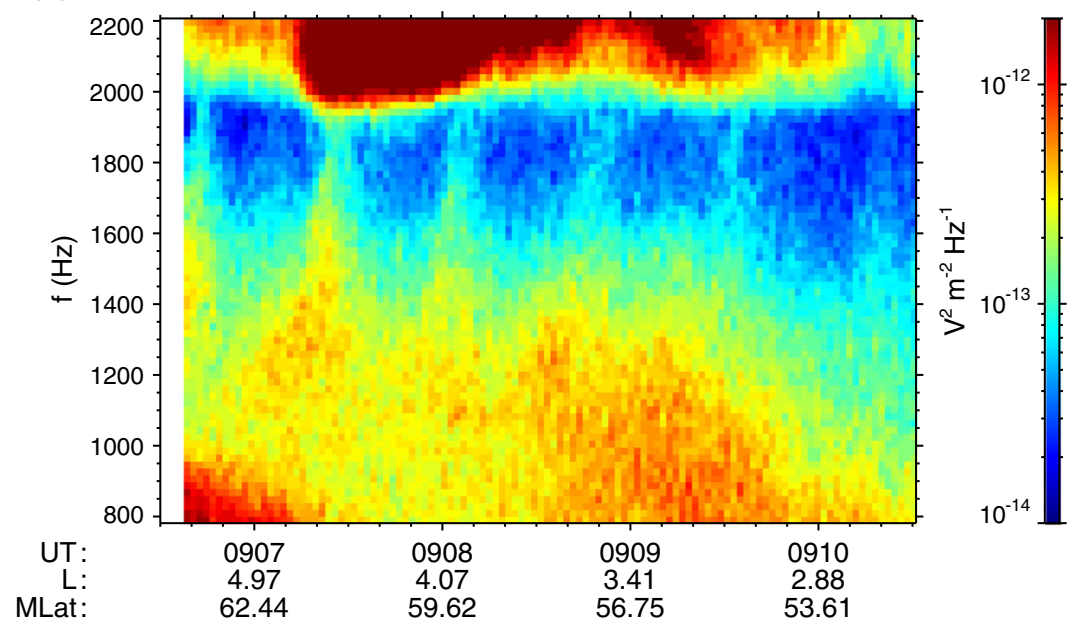

(b)

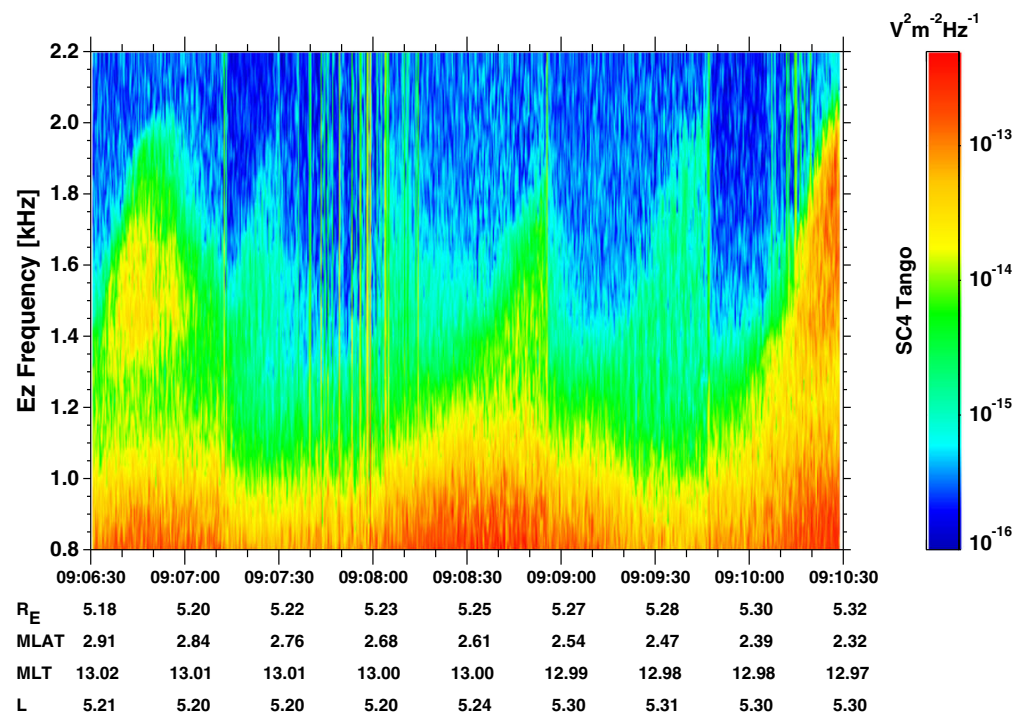

Figure 4. Frequency-time spectrograms of power spectral density of electric field fluctuations during the time interval when QP emissions were measured simultaneously by (a) DEMETER and (b) Cluster 4 on 13 April 2010. Both spacecraft observe the same QP structure. 
(a)

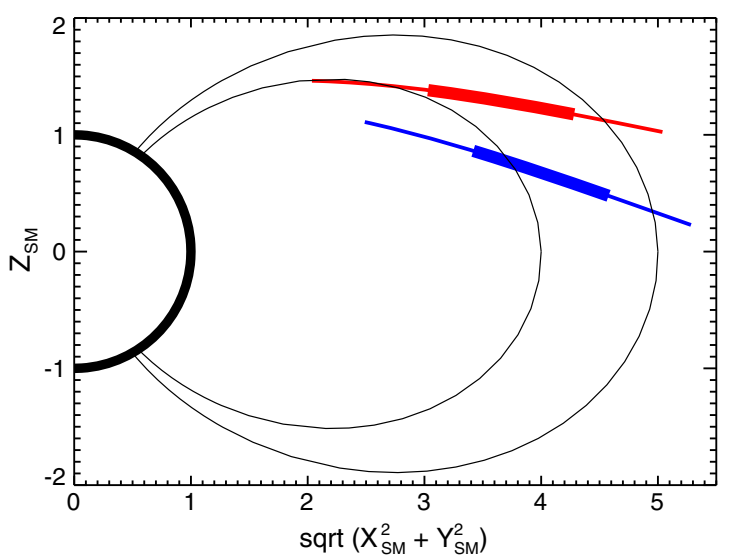

(b)

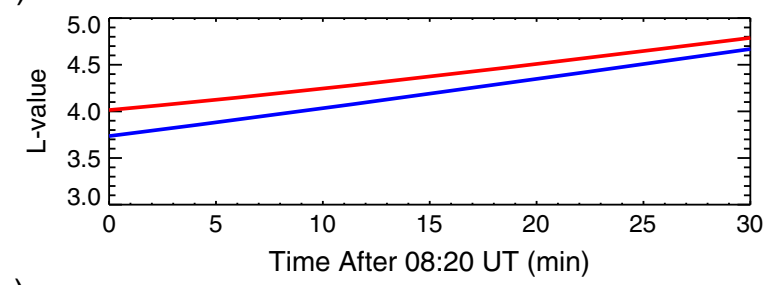

(c)

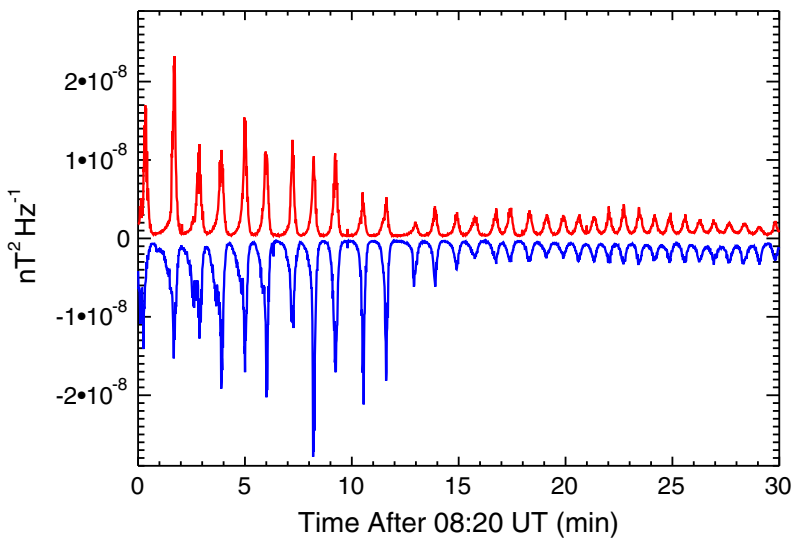

Figure 5. (a) Locations of Cluster 2 (red) and Cluster 4 (blue) spacecraft during the time interval when the QP emissions were observed by the STAFF-SA instruments on 13 April 2010. The parts of the orbits between 08:20 UT and 08:50 UT, which is the time interval used for a correlation analysis, are plotted in bold. Magnetic field lines corresponding to $L=4$ and $L=5$ calculated using combined IGRF and Tsyganenko 89 magnetic field models are overplotted. (b) $L$-values of Cluster 2 (red) and Cluster 4 (blue) during the time interval used for a correlation analysis. (c) Power spectral density of magnetic field fluctuations measured by the STAFF-SA instruments in the frequency range from about $1250 \mathrm{~Hz}$ up to $1575 \mathrm{~Hz}$. The values observed by Cluster 4 are plotted with a negative sign in order to enable an easy visual comparison with the values observed by Cluster 2.

results concerning the spatial extent of the QP event are shown in Figure 6. The black curves correspond to the DEMETER data, the red curve corresponds to the Cluster 2 data, and the blue curve corresponds to the Cluster 4 data. $L$-values and MLTs of the satellites between 04:30 UT and
09:30 UT are plotted in polar coordinates. The parts of the satellite orbits where QP emissions were observed are plotted in bold. Cluster spacecraft, coming from the dusk, started to see the QP emissions as soon as the WBD instruments turned on, i.e., as soon as the high-resolution wave measurements were available. However, the emissions faded out well before the end of the WBD measurements.

[14] The observations by Cluster performed close to the equatorial region clearly demonstrate that the QP emissions were observed at $L$-shells as large as $L \approx 5.5$. As for the lower boundary of the affected $L$-shells, the emissions were observed by Cluster at $L$-shells as low as $L \approx 3.25$. Moreover, DEMETER observations show that at low altitudes the QP emissions can be detected at $L$-shells as low as $L$ 1.5. Nevertheless, it is important to note that the reason why Cluster did not see the emissions at low $L$-values may be a limited MLT extent of the QP event. Namely, during the time interval when Cluster was located at low $L$-values, it was also at rather large MLTs. This limited MLT extent is in agreement with previous statistical studies, which showed that QP emissions occur predominantly on the dayside, with a maximum just before noon and another in the afternoon [Tixier and CornilleauWehrlin, 1986; Sazhin and Hayakawa, 1994]. The emissions were observed between about 7.4 and $12.3 \mathrm{~h}$ of MLT on DEMETER, while they spanned between about 12.5 and $14.8 \mathrm{~h}$ of MLT on Cluster. Assuming that the MLT extent of the event does not change over time, one can estimate that the MLT interval where the QP event occurred was at least about $7.5 \mathrm{~h}$.

[15] Previously published results of QP emissions have revealed that - at least in some cases-QP emissions were associated with corresponding ULF variations of the geomagnetic field [see, e.g., Sato et al., 1974; Sato and Fukunishi, 1981]. The fluxgate magnetometers on board the Cluster spacecraft allow us to check for the presence of magnetic field pulsations during the times when QP emissions are observed. Data from Cluster 2 and Cluster 4 are available for the time interval of interest. It is found that-apart from the expected variation due to the spacecraft motionULF magnetic field fluctuations are observed by Cluster 4 between about 08:50 UT and 09:10 UT. The amplitude of these fluctuations is depicted in Figure 7. The values plotted in the top and middle panels are the $B_{x}$ and $B_{y}$ components of the ULF fluctuations in the GSE coordinates, respectively. They were calculated from the measured magnetic field components by subtracting the $5 \mathrm{~min}$ boxcar average. No similar fluctuations occurred in the $B_{z}$ component of the magnetic field (not shown). Taking into account that during the time of observation Cluster 4 was located close to the equator, this means that the magnetic pulsations are approximately transverse to the ambient magnetic field. Interestingly, Cluster 2, which is located at comparable $L$-shells at geomagnetic latitudes larger by about $10^{\circ}$, observed only very weak magnetic pulsations limited to a short time interval close to 08:55 UT (not shown). The bottom panel of Figure 7 shows the frequency-time spectrogram of power spectral density of electric field fluctuations measured by the WBD instrument on board Cluster 4 for comparison. It can be seen that the period of the ULF magnetic field pulsations is of the same order, but somewhat longer than the period of QP elements. No exact one-to-one correlation between the observed magnetic pulsations and the QP elements has been found. 


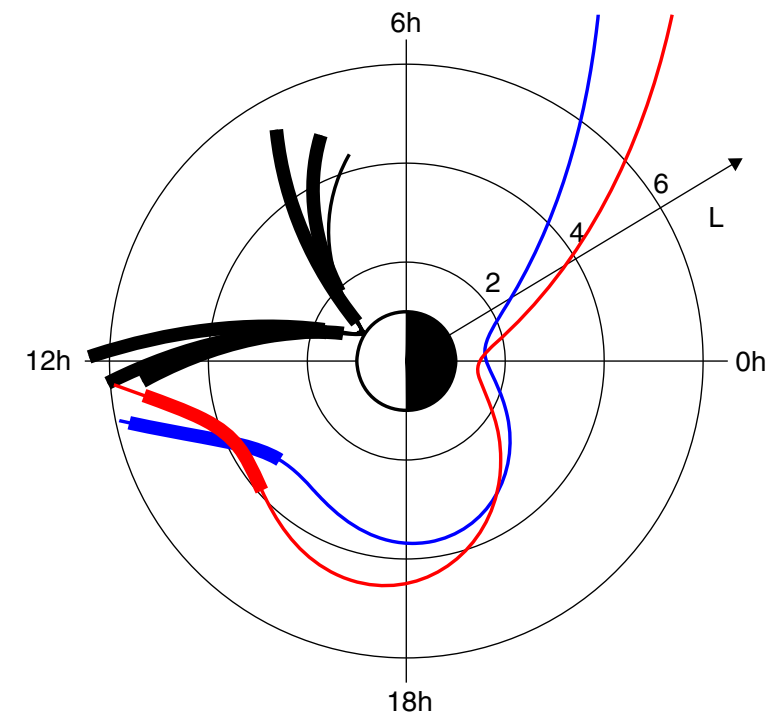

Figure 6. The extent of the analyzed QP event from 13 April 2010 as observed by DEMETER (black), Cluster 2 (red), and Cluster 4 (blue). $L$-values and MLTs of the satellites between 04:30 UT and 09:30 UT are plotted in polar coordinates. The parts of the satellite orbits where QP emissions were observed are plotted in bold. Cluster spacecraft, coming from the dusk, started to see the QP emissions as soon as the WBD instruments turned on. However, the emissions faded out well before the end of the WBD measurements.
[16] Multicomponent measurements of the electromagnetic field made by the STAFF-SA instruments on board the Cluster spacecraft allow us to perform a detailed wave analysis [see, e.g., Santolik et al., 2003, and references therein]. The results obtained for the time interval when the QP emissions were observed are shown in Figures 8 and 9 for Cluster 4 and Cluster 2, respectively. The top panel represents the power spectral density of magnetic field fluctuations. The second panel shows the results obtained for the planarity of polarization of the wave magnetic field obtained by a singular value decomposition (SVD) method. Values close to 1 correspond to the situation of a single plane wave, while values close to 0 correspond to the situation when the plane wave approximation becomes inappropriate. The third panel represents the ellipticity of magnetic field fluctuations obtained again by the SVD method. The absolute value of ellipticity is equal to the ratio of minor to major polarization axes, i.e., values close to 0 correspond to nearly linearly polarized waves and values close to 1 correspond to almost circularly polarized waves. The sign corresponds to the sense of polarization. A negative sign corresponds to lefthanded polarized waves, while a positive sign corresponds to right-handed polarized waves. The fourth panel represents the results obtained for the polar angles of the wave vector directions with respect to the ambient magnetic field determined using SVD of the magnetic parts of the spectral matrices. Taking symmetry into account, i.e., the fact that two antiparallel directions cannot be distinguished when using the magnetic field data only, the values of the polar angle
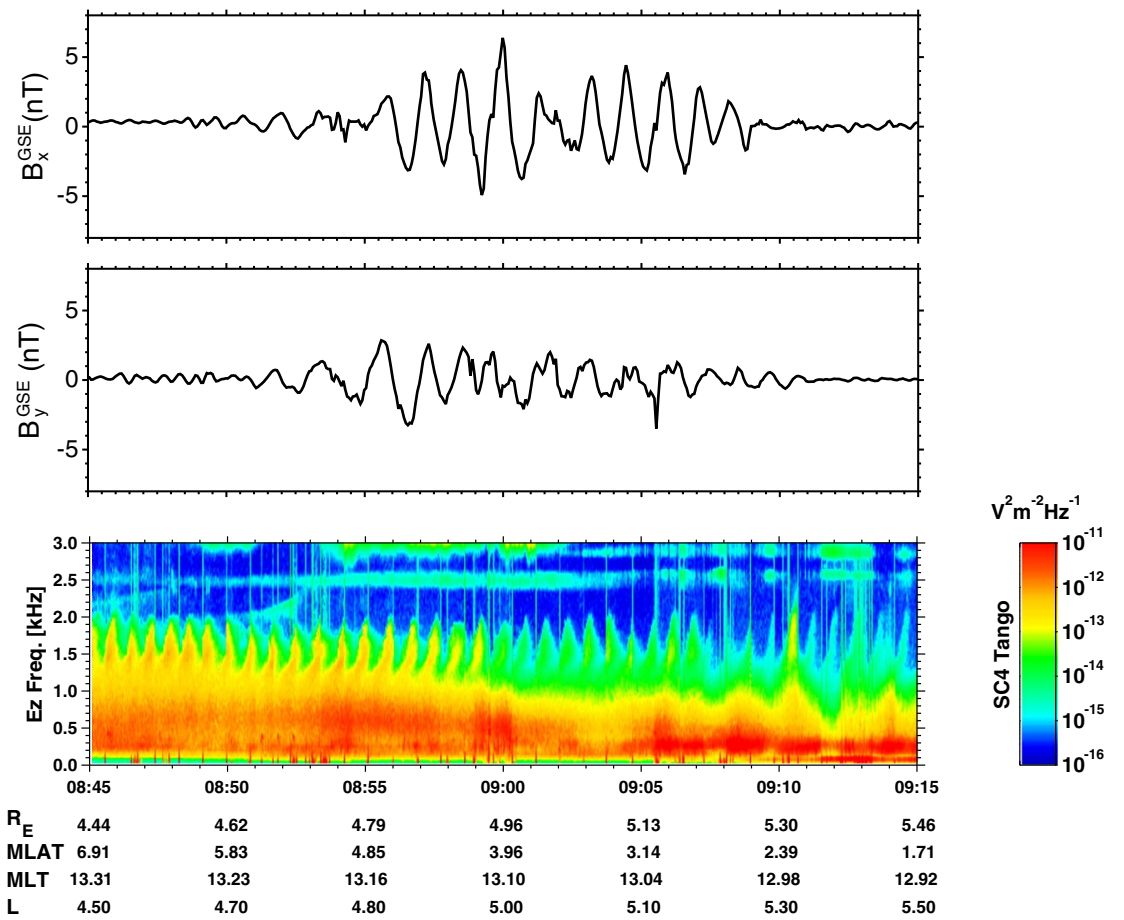

Figure 7. (top and middle panels) Magnetic field fluctuations measured by the FGM instrument on board Cluster 4 on 13 April 2010 around 09:00 UT, when the spacecraft was located close to the equatorial plane. (bottom) Frequency-time spectrogram of power spectral density of electric field fluctuations measured by the WBD instrument on board Cluster 4 during the same time. The period of the ULF magnetic field fluctuations is of the same order, but by a factor of about 1.6-1.7 larger than the period of QP emissions. 


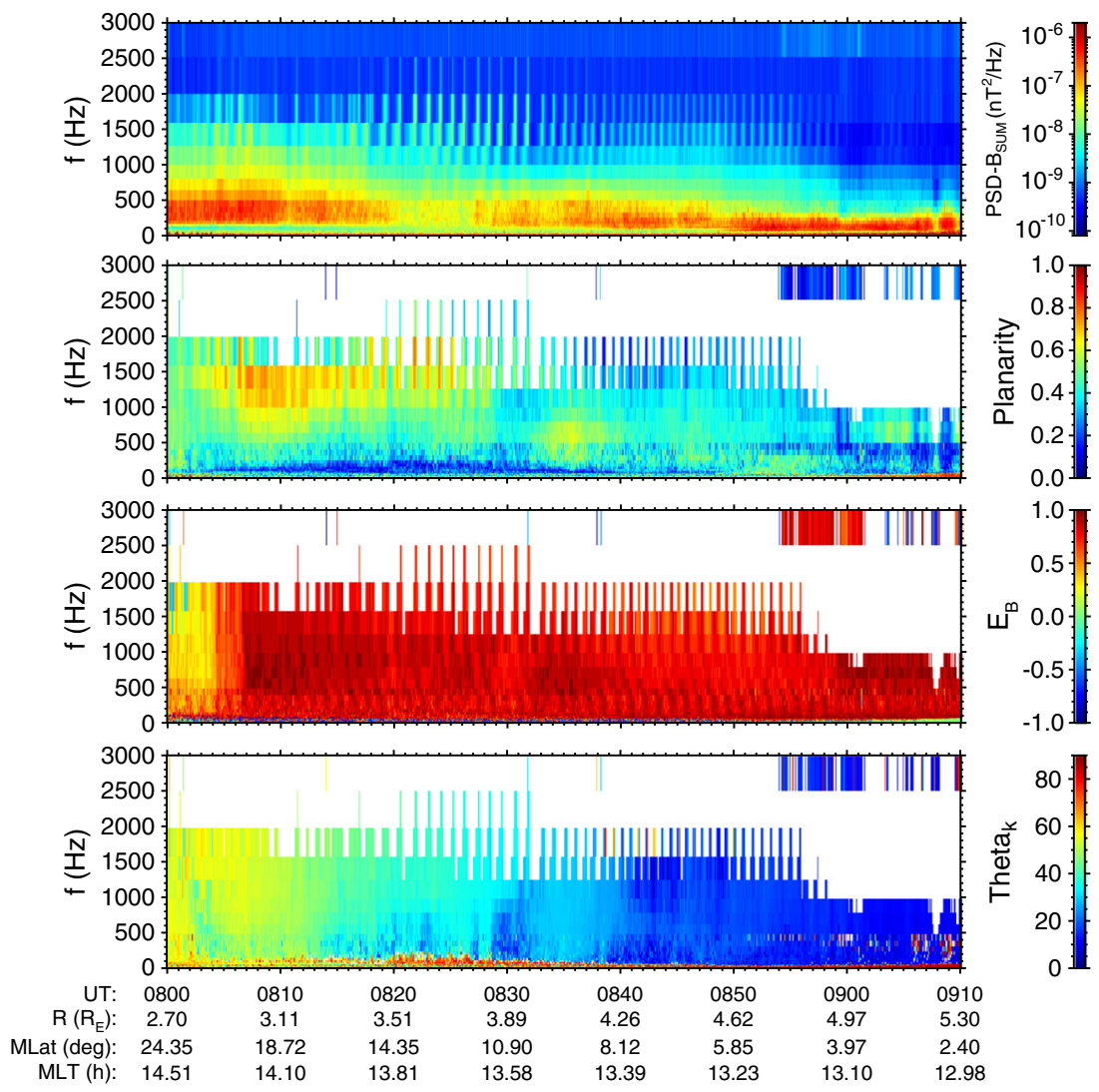

Figure 8. QP emissions from Figure 2 as observed by the STAFF-SA instrument on board Cluster 4 on 13 April 2010. The meaning of the individual panels is as follows: power spectral density of magnetic field fluctuations, planarity of magnetic field fluctuations, ellipticity of magnetic field fluctuations, and polar angle of the wave vector direction with respect to the ambient magnetic field.

range between $0^{\circ}$ and $90^{\circ}$. The values close to $0^{\circ}$ correspond to a nearly field-aligned propagation, while the values close to $90^{\circ}$ correspond to a nearly perpendicular propagation. The results of the wave analysis were calculated only for frequency-time intervals where the power spectral density of magnetic field fluctuations was larger than $8 \times 10^{-10} \mathrm{nT}^{2} \mathrm{~Hz}^{-1}$, in order not to perform the analysis for data intervals, which do not correspond to QP emissions.

[17] Several conclusions can be drawn from Figures 8 and 9. First, the planarity in the frequency-time intervals corresponding to QP emissions is generally rather low. This may either indicate that the Cluster spacecraft observe a superposition of several different waves coming from different directions, or it may be simply a result of rather lowpower spectral density of magnetic field fluctuations corresponding to the QP emissions. The emissions are right-handed and nearly circularly polarized. They are almost field-aligned close to the geomagnetic equator, but at higher geomagnetic latitudes the polar angle of the wave vector direction with respect to the ambient magnetic field gradually (for Cluster 4) or abruptly (Cluster 2) increases and the waves become more and more oblique.

\section{Discussion}

[18] The presented case study of QP emissions observed simultaneously on board the DEMETER and the Cluster spacecraft takes advantage of a unique satellite constellation.
Moreover, we were fortunate enough to have the WBD instruments on board Cluster 2 and Cluster 4 active during the time interval of interest. Although the obtained multipoint measurements are invaluable for our understanding of the properties of QP emissions, it is clear that on a longer run they need to be complemented by systematic surveys of QP emissions observed by DEMETER and Cluster separately. Such type of analysis will be done in the future. Our preliminary results based on visual identification of QP emissions in DEMETER data indicate that QP emissions are observed in about $3 \%$ of daytime half-orbits, while they are basically absent during the night. Note that we were likely to miss events with the modulation periods lower than about $10 \mathrm{~s}$ or the frequency bandwidth lower than about $400 \mathrm{~Hz}$, which however, taking into account the properties of QP emissions, should not prevent us from identifying most of them. Moreover, if the QP emissions occur, they are generally observed both in the beginning and at the end of the half-orbits, i.e., in two nearly geomagnetically conjugated areas.

[19] Simultaneous measurements at several well-separated points allow us to directly distinguish between spatial and temporal variations. The QP event of interest was observed by the STAFF-SA instruments on board Cluster 4 and Cluster 2 for a few tens of minutes (see Figures 8 and 9). During that time Cluster 2 was about $10^{\circ}$ of geomagnetic latitude to the North from Cluster 4, being separated by about $0.5 \mathrm{~h}$ of MLT and located at slightly lower radial distances. 


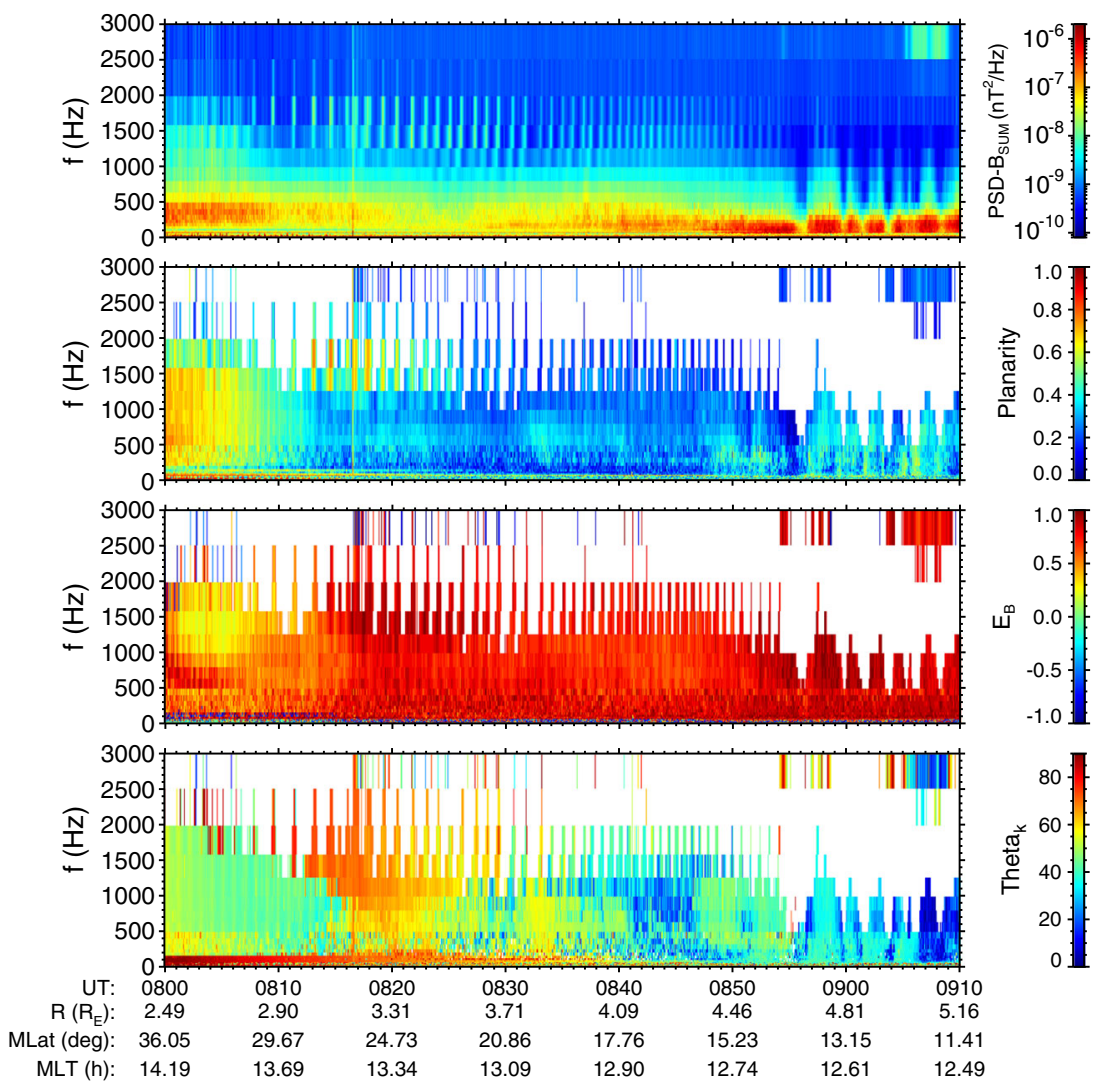

Figure 9. QP emissions observed by the STAFF-SA instrument on board Cluster 2 on 13 April 2010. The meaning of individual panels is the same as in Figure 8.

Nevertheless, the observed QP modulation was - within the experimental error - the same (see Figure 5). Similarly, the same QP modulation was observed on board the Cluster 4 and the DEMETER spacecraft during the time when the QP event was detected by both of them (Figure 4). This clearly indicates that during the time duration of the event the same QP modulation of the wave intensity is observed at very different locations. This is in agreement with the results obtained by Morrison et al. [1994] and Engebretson et al. [2004], who reported that QP emissions observed simultaneously at two or more ground-based stations are synchronized within the $1 \mathrm{~s}$ time resolution of the samples. However, the observations on the ground might have been strongly affected by the wave propagation in the Earth-ionosphere waveguide. Consequently, these authors have suggested that the emissions were likely to be generated in, and propagate along, a particular duct. The identical signals observed at great distances were then explained by the signal penetrating the ionosphere at the base of the duct and propagating in the Earth-ionosphere waveguide. It is clear that such a scheme cannot explain our satellite-based results of the same QP modulation being observed at several different points in space.

[20] Nevertheless, this finding might be possibly explained by the suggested generation mechanism based on ULF compressional waves propagating close to the equatorial plane [Kimura, 1974; Sato and Fukunishi, 1981], because such a scheme naturally allows for waves with the same modulation to be generated over a significant area of the equatorial plane.
In this sense the generation mechanism might be similar to the mechanism suggested by Němec et al. [2012] to explain the properties of a large-scale Magnetospheric Line Radiation event observed simultaneously on board the Cluster 1, Cluster 2, and DEMETER spacecraft. Moreover, one should consider that the QP emissions may propagate from the generation region at oblique wave normal angles, as is suggested by the higher-latitude results depicted in the bottom panels of Figures 8 and 9. The ducted-nonducted mode conversion process may provide a means by which signals generated, triggered, or amplified in small localized ducts can spread into much larger regions of the inner magnetosphere [Smith et al., 1984]. Moreover, raytracing studies suggest that if guiding provided by the plasmapause is considered, the waves deviate toward lower $L$-values at altitudes of a few thousands of kilometers [Inan and Bell, 1977]. This may possibly help to explain why the QP emissions extend down to very low $L$-shells on DEMETER (see Figure 6).

[21] Concerning the azimuthal extent of the QP event, Figure 6 shows that the DEMETER satellite observed the event at MLTs from about $7.4 \mathrm{~h}$ up to about $12.3 \mathrm{~h}$. The Cluster spacecraft observed the event at the very end of the analyzed time interval, spanning the MLTs from about 12.5 up to $14.8 \mathrm{~h}$. Assuming that the generation region of the QP emissions is located at a fixed MLT, this would correspond to the source spanning over as much as $7.5 \mathrm{~h}$ in MLT. Moreover, the limited extent of the QP emissions in MLT is the most probable explanation for why Cluster did not see them before about 08:00 UT. 
[22] ULF magnetic field fluctuations detected by Cluster 4 close to the equatorial region and depicted in Figure 7 correspond to the shear Alfvén wave with the wave vector parallel to the ambient magnetic field. The period of the ULF oscillations is larger than the period of the QP emissions by a factor of about 1.6-1.7. However, the mechanisms considered for the generation of QP emissions based on the modulation of VLF waves by geomagnetic pulsations generally involve compressional fluctuations of the magnetic field with the same period as the period of QP emissions [Kimura, 1974]. It is thus possible that the observed ULF magnetic field fluctuations do not correspond to the modulating ULF wave responsible for the QP generation itself, but rather to the excited standing oscillations of local resonant field lines [Sato and Fukunishi, 1981; Tixier and Cornilleau-Wehrlin, 1986]. In any case, the fluctuations seem to be limited to the near-equatorial region, as they are observed by Cluster 4 located close to the equatorial plane, while Cluster 2 located at geomagnetic latitudes by $10^{\circ}$ larger principally fails to see them. This would be consistent with field line oscillations at small even harmonics [see Figure 9 of Takahashi and McPherron, 1982].

[23] Particle measurements that would allow us to determine the position of the plasmapause are not available. Nevertheless, the measurements by the Whisper instrument [Décréau et al., 2001] show that on its inbound path Cluster 2 did not encounter the plasmasphere before about 06:38 UT and Cluster 4 did not encounter the plasmasphere before about 06:26 UT, which corresponds to $L$-values of about 4.8 and 4.4, respectively. Assuming that the location of the plasmapause did not change significantly during $2-3 \mathrm{~h}$ between these measurements and the time interval of interest, one can consider these $L$-values as the upper estimates of the plasmapause location. Such a result is quite in agreement with the empirical model by Carpenter and Anderson [1992], which gives $L_{\mathrm{pp}} \approx 4.2$. Consequently, the ULF magnetic fluctuations detected by Cluster 4 close to the equatorial plane seem to be located outside the plasmasphere, but rather close to its outer boundary. As for their limited time duration, it might be appealing to identify their beginning with the Cluster 4 crossing of the plasmapause. They disappear at about the same time as the QP emissions fade, indicating that the two are indeed most probably connected. Unfortunately, the QP emissions disappear before Cluster 4 crosses the geomagnetic equator, so that the data from this specific region of extreme interest are not available.

[24] The results of a detailed wave analysis presented in Figures 8 and 9 are - to the best of our knowledge - the first measurements of propagation and polarization properties of QP emissions close to their probable source region. The second panels of the figures show that the planarity of polarization of the wave magnetic field is generally very low, corresponding either to a situation of several concurrent electromagnetic waves propagating from different directions, or to a low signal-to-noise ratio. It is not possible to distinguish between these two possibilities using the available experimental data. However, given the low power spectral density of magnetic field fluctuations corresponding to the QP emissions, the latter explanation seems to be more likely. Most importantly, the QP emissions appear to be nearly field-aligned close to the geomagnetic equator and they become more and more oblique with increasing geomagnetic latitude. This indicates that their propagation is primarily unducted.

\section{Conclusions}

[25] Results of a case study of QP emissions observed simultaneously by the Cluster spacecraft close to the equatorial region at $L$-values of about 3.3-5.5 and by the lowaltitude DEMETER spacecraft have been presented. The analyzed QP event lasted as long as $5 \mathrm{~h}$. Conjugate observations at several different points in space allowed us to demonstrate that principally the same modulation pattern is observed at $L$-shells from about 1.5 to 5.5 and over several hours of MLT. This finding is particularly important, because the observations are done in space and cannot be therefore explained by propagation in the Earth-ionosphere waveguide, contrary to what was suggested by Morrison [1990] and Engebretson et al. [2004]. Instead of that, the same observed QP modulation must be linked to the generation mechanism itself and/or the unducted propagation of QP emissions in the magnetosphere. This is further supported by the results of a detailed wave analysis, which show that the QP emissions are probably generated in the equatorial region (propagation parallel to the ambient magnetic field), and at higher geomagnetic latitudes they propagate at oblique wave normal angles. ULF magnetic field fluctuations with a period roughly comparable to, but somewhat larger than the period of the QP modulation were detected by the Cluster spacecraft. They appeared to be limited to the vicinity of the geomagnetic equator and were polarized principally in the equatorial plane.

[26] Acknowledgments. We would like to thank the Cluster Active Archive and all the involved personnel. We would like to acknowledge E. Lucek, who is the principal investigator of the FGM instrument, J.-G. Trotignon, who is the principal investigator of the Whisper instrument, and all their teams. The used Cluster WBD data were received at the Panska Ves Observatory and we would like to acknowledge all the responsible personnel. DEMETER was operated by CNES and the corresponding staff is deeply acknowledged. We also thank J. J. Berthelier, who is the principal investigator of the electric field instrument. This work was supported by GACR grants P209/12/P658, P205/10/2279, and P209/11/2280. This work was supported at Iowa by NASA Goddard Space Flight Canter grant NNX11AB38G.

\section{References}

Alford, J., M. Engebretson, R. Arnoldy, and U. Inan (1996), Frequency variations of quasi-periodic ELF-VLF emissions: A possible new groundbased diagnostic of the outer high-latitude magnetosphere, J. Geophys. Res., 101(A1), 83-97.

Balogh, A., et al. (1997), The Cluster magnetic field investigation, Space Sci. Rev., 79, 65-91.

Balogh, A., et al. (2001), The Cluster magnetic field investigation: an overview of in-flight performance and initial results, Ann. Geophys., 19, 1207-1217.

Bell, T. F. (1976), ULF wave generation through particle precipitation induced by VLF transmitters, J. Geophys. Res., 81(19), 3316-3326.

Berthelier, J. J., et al. (2006), ICE, the electric field experiment on DEMETER, Planet. Space Sci., 54, 456-471.

Carpenter, D. L., and R. R. Anderson (1992), An ISEE/Whistler model of equatorial electron density in the magnetosphere, J. Geophys. Res., 97(A2), 1097-1108.

Carson, W. B., J. A. Koch, J. H. Pope, and R. M. Gallet (1965), Long-period very low frequency emission pulsations, J. Geophys. Res., 70(17), 4293-4303.

Chen, L. (1974), Theory of ULF modulation of VLF emissions, Geophys. Res. Lett., 1(2), 73-75.

Cornilleau-Wehrlin, N., et al. (1997), The Cluster spatio-temporal analysis of field fluctuations (STAFF) experiment, Space Sci. Rev., 79(1-2), doi:10.1023/A:1004979209565. 


\section{NĚMEC ET AL.: QUASI-PERIODIC EMISSIONS}

Cornilleau-Wehrlin, N., et al. (2003), The Cluster spatio-temporal analysis of field fluctuations (STAFF) experiment, Ann. Geophys., 21, 437-456.

Coronity, F. V., and C. F. Kennel (1970), Electron precipitation pulsations, J. Geophys. Res., 75(7), 1279-1289.

Décréau, P. M. E., et al. (2001), Early results from the Whisper instrument on Cluster: an overview, Ann. Geophys., 19, 1241-1258.

Engebretson, M. J., J. L. Posch, A. J. Halford, G. A. Shelburne, A. J. Smith, M. Spasojević, U. S. Inan, and R. L. Arnoldy (2004), Latitudinal and seasonal variations of quasiperiodic and periodic VLF emissions in the outer magnetosphere, J. Geophys. Res., 109(A05216), doi:10.1029/2003JA010335.

Gendrin, R., C. Berthomier, H. Cory, A. Meyer, B. Sukhera, and J. Vigneron (1970a), Very-low-frequency and particle rocket experiment at Kerguelen Islands: 1. very-low-frequency measurements, J. Geophys. Res., 75(31), 6153-6168.

Gendrin, R., J. Etcheto, and B. de la Porte des Vaux (1970b), Very-lowfrequency and particle rocket experiment at Kerguelen Islands: 2. particle measurements, J. Geophys. Res., 75(31), 6169-6181.

Gurnett, D. A., R. L. Huff, and D. L. Kirchner (1997), The wide-band plasma wave investigation, Space Sci. Rev., 79, 195-208.

Helliwell, R. A. (1965), Whistlers and related ionospheric phenomena, Stanford Univ. Press, CA.

Ho, D. (1973), Interaction between whistlers and quasi-periodic VLF emissions, J. Geophys. Res., 78(31), 7347-7356.

Inan, U. S., and T. F. Bell (1977), The plasmapause as a VLF wave guide, J. Geophys. Res., 82(19), 2819-2827.

Kimura, I. (1974), Interrelation between VLF and ULF emissions, Space Sci. Rev., 16, 389-411.

Kitamura, T., J. A. Jacobs, T. Watanabe, and J. R. B. Flint (1969), An investigation of quasi-periodic VLF emissions, J. Geophys. Res., 74(24), 5652-5664.

Lanzerotti, L. J., C. G. Maclennan, L. V. Medford, and D. L. Carpenter (1986), Study of a QP/GP event at very high latitudes, J. Geophys. Res., 91(A1), 375-380.

Morrison, K. (1990), Quasi-periodic VLF emissions and concurrent magnetic pulsations seen at 1=4, Planet. Space Sci., 38(12), 1555-1565.

Morrison, K., M. J. Engebretson, J. R. Beck, J. E. Johnson, R. L. Arnoldy, J. L. J. Cahill, D. L. Carpenter, and M. Gallani (1994), A study of quasiperiodic ELF-VLF emissions at three antarctic stations: Evidence for off-equatorial generation?, Ann. Geophys., 12, 139-146, doi:10.1007/ s00585-994-0139-8.

Němec, F., M. Parrot, O. Santolík, and J. S. Pickett (2012), Magnetospheric line radiation event observed simultaneously on board Cluster 1, Cluster 2 and DEMETER spacecraft, Geophys. Res. Lett., 39(L18103), doi:10.1029/2012GL053132.

Pasmanik, D. L., E. E. Titova, A. G. Demekhov, V. Y. Trakhtengerts, O. Santolik, F. Jiricek, K. Kudela, and M. Parrot (2004), Quasi-periodic
ELF/VLF wave emissions in the Earth's magnetosphere: Comparison of satellite observations and modelling, Ann. Geophys., 22, 4351-4361.

Santolík, O., M. Parrot, and F. Lefeuvre (2003), Singular value decomposition methods for wave propagation analysis, Radio Sci., 38(1), doi:10.1029/2000RS002523.

Sato, N., and H. Fukunishi (1981), Interaction between ELF-VLF emissions and magnetic pulsations: Classification of quasi-periodic ELF-VLF emissions based on frequency-time spectra, J. Geophys. Res., 86(A1), 19-29.

Sato, N., and S. Kokubun (1980), Interaction between ELF-VLF emissions and magnetic pulsations: Quasi-periodic ELF-VLF emissions associated with Pc 3-4 magnetic pulsations and their geomagnetic conjugacy, J. Geophys. Res., 85(A1), 101-113.

Sato, N., and S. Kukubun (1981), Interaction between ELF-VLF emissions and magnetic pulsations: Regular period ELF-VLF pulsations and their geomagnetic conjugacy, J. Geophys. Res., 86(A1), 9-18.

Sato, N., and T. Matsudo (1986), Origin of magnetic pulsations associated with regular period VLF pulsations (Type $2 \mathrm{QP}$ ) observed on the ground at syowa station, J. Geophys. Res., 91(A10), 11,179-11,185.

Sato, N., K. Hayashi, S. Kokubun, T. Oguti, and H. Fukunishi (1974), Relationships between quasi-periodic VLF emission and geomagnetic pulsation, J. Atm. and Terr. Phys., 36, 1515-1526.

Sazhin, S. S. (1987), An analytical model of quasiperiodic ELF-VLF emissions, Planet. Space Sci., 35(10), 1267-1274.

Sazhin, S. S., and M. Hayakawa (1994), Periodic and quasiperiodic VLF emissions, J. Atm. and Terr. Phys., 56(6), 735-753.

Smith, A. J., D. L. Carpenter, and U. S. Inan (1984), Whistler-triggered VLF noise bursts observed on the DE-1 satellite and simultaneously at antarctic ground stations, Ann. Geophys., 3(1), 81-88.

Smith, A. J., D. L. Carpenter, Y. Corcuff, J. P. S. Rash, and E. A. Bering (1991), The longitudinal dependence of whistler and chorus characteristics observed on the ground near $l=4$, J. Geophys. Res., 96(A1), 275-284, doi:10.1029/90JA01077.

Smith, A. J., M. J. Engebretson, E. M. Klatt, U. S. Inan, R. L. Arnoldy, and H. Fukunishi (1998), Periodic and quasiperiodic ELF/VLF emissions observed by an array of Antarctic stations, J. Geophys. Res., 103(A10), 23,611-23,622.

Takahashi, K., and R. L. McPherron (1982), Harmonic structure of Pc 3-4 pulsations, J. Geophys. Res., 87(A3), 1504-1516.

Tixier, M., and N. Cornilleau-Wehrlin (1986), How are the VLF quasiperiodic emissions controlled by harmonics of field line oscillations? the results of a comparison between ground and GEOS satellites measurements, J. Geophys. Res., 91(A6), 6899-6919.

Watt, C. E. J., A. W. Degeling, R. Rankin, K. R. Murphy, I. J. Rae, and H. J. Singer (2011), Ultralow-frequency modulation of whistler-mode wave growth, J. Geophys. Res., 116(A10209), doi:10.1029/2011JA016730. 